\title{
Circadian clock of Aedes aegypti: effects of blood-feeding, insemination and RNA interference
}

\author{
Carla Gentile', Gustavo Bueno da S Rivas ${ }^{1}$, José BP Lima ${ }^{2,3,4}$, \\ Rafaela Vieira Bruno ${ }^{1,2} /+$, Alexandre Afranio Peixoto ${ }^{1,2,+}$ \\ 'Laboratório de Biologia Molecular ${ }^{3}$ Laboratório de Fisiologia e Controle de Artrópodes Vetores, Instituto Oswaldo Cruz-Fiocruz, \\ Rio de Janeiro, RJ, Brasil ' Instituto Nacional de Ciência e Tecnologia em Entomologia Molecular, Rio de Janeiro, RJ, Brasil \\ ${ }^{4}$ Instituto de Biologia do Exército, Rio de Janeiro, RJ, Brasil
}

\begin{abstract}
Mosquitoes are the culprits of some of the most important vector borne diseases. A species' potential as a vector is directly dependent on their pattern of behaviour, which is known to change according to the female's physiological status such as whether the female is virgin/mated and unfed/blood-fed. However, the molecular mechanism triggered by and/or responsible for such modulations in behaviour is poorly understood. Clock genes are known to be responsible for the control of circadian behaviour in several species. Here we investigate the impact mating and blood-feeding have upon the expression of these genes in the mosquito Aedes aegypti. We show that blood intake, but not insemination, is responsible for the down-regulation of clock genes. Using RNA interference, we observe a slight reduction in the evening activity peak in the fourth day after dstim injection. These data suggest that, as in Drosophila, clock gene expression, circadian behaviour and environmental light regimens are interconnected in Ae. aegypti.
\end{abstract}

Key words: clock genes - blood-feeding - insemination - RNAi - behaviour - Aedes aegypti

Daily rhythmic behaviours of living organisms are endogenously sustained by the circadian clock - a series of molecular transcriptional/translational negative feedback loops with cycles of about $24 \mathrm{~h}$. It is known from Drosophila melanogaster that this system is mainly composed of the clock genes period (per), timeless (tim), cycle $(c y c)$ and Clock $(C l k)$, that encode their respective proteins PER, TIM, CYC and CLK. The latter two proteins form a heterodimer that activates the transcription of per and tim. In turn, PER and TIM heterodimerise and enter the nucleus to inhibit CYC:CLK, thereby repressing their own transcription [reviewed in Hardin (2011)]. Regulatory steps mean this cycle takes about $24 \mathrm{~h}$ to be completed. Although it is defined as self-sustainable, the circadian clock is sensitive to external cues of passing time in order to keep the organism in fine tune with its environment. The best understood external input to the clock is light. It acts upon a light-sensitive protein called CRYPTOCHROME that triggers the earlier degradation of TIM, resetting the system (Emery et al. 1998, Stanewsky et al. 1998, Ceriani et al. 1999). Although this molecular feedback loop can be found in several tissues of the fly, the master control of this inner clock, respon-

doi: 10.1590/0074-0276130471

Financial support: CNPq, FAPERJ, HHMI, FIOCRUZ

CG current address: Neuroscience Program, Garvan Institute of Medical Research, Darlinghurst, Sydney, New South Wales, Australia

$\dagger$ In memoriam

+ Corresponding author: rafaelav@ioc.fiocruz.br

Received 24 July 2013

Accepted 29 November 2013 sible for orchestrating outputs such as activity/rest behaviour, lies in certain groups of neurons in the brain known as clock neurons [reviewed in Hardin (2011) and Hardin and Panda (2013)].

Previous studies, reported in a number of other insect species, have broadened our understanding of how clock genes operate and relate to a greater variety of behaviours [reviewed in Sandrelli et al. (2008)]. Haematophagy is a relevant feature of some arthropods' behaviour since it is the primary mechanism through which diseases are transmitted to humans and/or other animals (Lehane 2005). Several studies conducted both in the field and in the laboratory have shown that the circadian activity of the mosquito female switches according to its physiological status (e.g., virgin vs. inseminated or unfed vs. blood-fed) and in different ways depending on the ecology of the species [reviewed in Clements (1999)]. These observations have encouraged investigations on the role mating and haematophagy could play in modulating clock gene expression in blood-feeding insects. Studies conducted in the sandfly Lutzomyia longipalpis (Meireles-Filho et al. 2006) and in the mosquito Anopheles gambiae (Das \& Dimopoulos 2008, Rund et al. 2011, 2013) have shown that clock gene expression and several clock-controlled genes' expression might be affected by blood intake. In the present study, we investigated the effect of blood-feeding and insemination on the expression of the main clock genes per, tim, cyc and Clk, in the head and body of the female Aedes aegypti mosquito - vector of dengue and yellow fever viruses (Consoli \& Lourenço-de-Oliveira 1994). In addition, we used RNAi to knockdown the expression of tim and investigate its effects on the pattern of Ae. aegypti activity behaviour under different light conditions. 


\section{MATERIALS AND METHODS}

Mosquitoes - The mosquitoes used in this study were from a laboratory colony of Ae. aegypti (Rockefeller strain) maintained at Institute of Biology of the Brazilian Army, state of Rio de Janeiro, Brazil. For all experiments, they were pre-synchronised from pupa stage to $12 \mathrm{~h}$ of light and $12 \mathrm{~h}$ of dark (LD 12:12) at $25^{\circ} \mathrm{C} \pm 0.5^{\circ} \mathrm{C}$ in a Precision Scientific Incubator Mod. 818 and newly emerged females were separated from males while still virgins and maintained at the same conditions.

For experiments with blood-fed females, four-fivedays old females were fed on anesthetised Guinea pig (ketamine: xylazine $80-120: 10-16 \mathrm{mg} / \mathrm{kg}$ ) for $1 \mathrm{~h}$, according to institutional procedures, oriented by the national guideline The Brazilian Legal Framework on the Scientific Use of Animals (Filipecki et al. 2011).

Samples for blood-feeding and insemination experiments - Virgin females were initially separated in two groups of 200 individuals. To one group we added 300 males for insemination for two days; the other group remained virgin. All mosquitoes were provided with $10 \%$ glucose solution and placed under the same conditions used to synchronise the pupae. The inseminated females were separated from the males and split into two groups of 80 females. Virgin females were also split in two groups of 80 individuals. On the same day, $5 \mathrm{~h}$ after lights-on or at Zeitgeber-time (ZT) 5 , one cage of virgin females and one cage of inseminated females were provided with a source of blood meal (guinea pig) for $1 \mathrm{~h}$. After this procedure, there were four distinct groups of Ae. aegypti females: (i) virgins/unfed, (ii) inseminated/unfed, (iii) virgins/blood-fed and (iv) inseminated/blood-fed. To allow one day entrainment after blood meal, we collected the females at ZT11 of the following day (11 $\mathrm{h}$ after lights-on and $\sim 30 \mathrm{~h}$ after the blood meal), a time point of the average expression of most clock genes (Gentile et al. 2009). All mosquitoes were frozen in liquid nitrogen and each of the four groups was separated into five samples of 10 individuals each. In the case of the two blood-fed groups, only females with visual evidence of blood intake (colour and volume of the abdomen) were considered. We beheaded the mosquitoes on a metal plate placed on dry ice to ensure RNA integrity. Therefore, the five replicas of each group of females generated two sub-samples: (i) 10 heads and (ii) 10 beheaded bodies. All these experiments were repeated four times.

RNA interference (RNAi) experiments - We used the RNAi technique to achieve the knockdown of tim expression and evaluate its effects on the activity of Ae. aegypti. The first $800 \mathrm{bp}$ of the coding sequence of Ae. aegypti tim gene (Gentile et al. 2006) were amplified and cloned into pGEM-T Easy vector (Promega) according to the manufacturer's protocol. The plasmid containing the cloned tim sequence fragment was used in a polymerase chain reaction (PCR) with oligonucleotides complementary to tim and containing the sequence of T7 promoter (underlined) in their 5' end: 5'-TAATACGACTCACTATAGGGAGAAGCGGAGAAGGATGGAATG-3' (forward primer) and 5'-TAATACGACTCACTATAGGGAGAT-
GGAAGGAAGTGTTATGATTGG-3' (reverse primer), generating an amplicon of about $590 \mathrm{bp}$. The product of the reaction was purified with MicroSpin S-400 HR Columns (GE Healthcare Life Sciences) and used in the synthesis of double-stranded RNA (dsRNA) with MegaScript (Life Technologies), according to the manufacturer's protocol. The dsRNA was purified with MegaClear (Life Technologies) and quantified in a spectrophotometer. The same procedure was conducted to generate a dsRNA of $\mathrm{LacZ}$ gene, used as a control, using the primers 5'-TAATACGACTCACTATAGGGAGAATCCGACGGGTTGTTACT-3' (forward) and 5'-TAATACGACTCACTATAGGGAGACACCACGCTCATCGATAATTT-3' (reverse).

The dsRNA was injected at a concentration of $3 \mu \mathrm{g} /$ $\mu \mathrm{L}$ into two-three days old virgin Ae. aegypti females using FemtoJet micro-injector (Eppendorf), adjusted to 200 hectoPascal and for $0.5 \mathrm{sec}$. The mosquitoes were temporarily paralysed by cooling, placed on a metal plate (on ice) and injected in the mesothorax with the dsRNA solution (Garver \& Dimopoulos 2007). Four hundred virgin females were injected, half with dsRNA for tim (dstim) and half with dsRNA for LacZ (dsLacZ). After the injection, the mosquitoes were transferred to cages provided with $10 \%$ glucose solution and placed in the Precision Scientific Incubator (Mod. 818) under LD 12:12 and $25 \mathrm{C} \pm 0.5^{\circ} \mathrm{C}$. After a couple of hours of recovery, 32 females from each of the four groups were prepared for the behavioural assay, while the remaining mosquitoes were kept in the incubator under the same conditions used for the behavioural assay and later collected for gene expression analysis.

Two independent experiments (called "LD" and "DD") were performed, differing on the light regime in which the females were kept after the injections. In the LD experiment, the same regime of the pre-synchronization of LD 12:12 for seven days was used; in the DD experiment they were kept under LD 12:12 for two days followed by constant darkness for five days. The samples for gene expression analysis were collected in five replicas with four mosquitoes each on the fourth day after the injection, on the ZT11 in the LD experiment (11th h after lights-on) and on the circadian time 11 (corresponding to the 11th $\mathrm{h}$ of the "subjective" day) in the DD experiment. As before, mosquitoes were beheaded on a metal plate and placed on dry ice to ensure RNA integrity, generating independent sub-samples of heads and beheaded bodies. Gene quantification was performed with quantitative real-time PCR (qRT-PCR).

RNA extractions and cDNA synthesis - Total RNA was extracted with $1 \mathrm{~mL}$ TRIzol (Life Technologies), purified with lithium chloride $2.5 \mathrm{M}$ and resuspended in RNAse-free water. cDNA was synthesised with TaqMan Reverse Transcription Reagents (Life Technologies) using oligo-dT in a final volume of $20 \mu \mathrm{L}$, according to the manufacturer's protocol and temperature conditions of $25^{\circ} \mathrm{C}$ for $10 \mathrm{~min}, 48^{\circ} \mathrm{C}$ for $1 \mathrm{~h}$ and $95^{\circ} \mathrm{C}$ for $5 \mathrm{~min}$. The cDNA used for qPCR assays was diluted in mili-Q water in a 1:9 proportion.

$q R T-P C R$ - Relative quantification of gene expression was conducted using qRT-PCR, performed in ABI Prism 
7000 Sequence Detection Systems (Applied Biosystems) and using Power SYBR Green PCR Master Mix (Life Technologies), oligonucleotides at a final concentration of $0.5 \mu \mathrm{M}$ and $4.0 \mu \mathrm{L}$ of cDNA, in a final reaction volume of $20 \mu \mathrm{L}$. Reactions were performed in four replicas for each sample and independently for the clock genes per, tim, cyc and Clk plus the endogenous control rp49, using the same oligonucleotides designed for a previous work (Gentile et al. 2009). The relative quantification of the gene expression was determined using the comparative Cycle threshold (Ct) method, also known as the $\Delta \Delta \mathrm{Ct}$ or 2- ${ }^{-\Delta \mathrm{Ct}}$ (Pfaffl 2001). Analysis of the data was carried out using Microsoft Excel software. For more details see previous publications (Gentile et al. 2006, 2009).

Behavioural analysis - Circadian locomotor activity rhythms of Ae. aegypti were recorded automatically using a larger version of the Drosophila Activity Monitoring system (Trikinetics) as previously described (Gentile et al. 2009, Lima-Camara et al. 2011), in a Precision Scientific Incubator Mod. 818. Two independent behavioural experiments were conducted: one under LD 12:12 for seven days (LD experiment) and the other under LD 12:12 for two days followed by constant darkness for five days (DD experiment). Temperature was kept constant at all times at $25^{\circ} \mathrm{C} \pm 0.5^{\circ} \mathrm{C}$. We used ActogramJ to analise the actograms and the $\chi^{2}$ periodograms (Schmid et al. 2011).

Statistical analysis - The Student $t$ test and ANOVA analysis were performed using SPSS v.17 (IBM ${ }^{\odot} 2008$, Chicago, USA).

\section{RESULTS}

Effect of blood-feeding and insemination on clock gene expression - We tested the effect of blood-feeding and insemination on the expression of the clock genes per, tim, cyc and Clk, both in the head and body of Ae. aegypti females, comparing four groups of mosquitoes in distinct physiological states: (i) virgin/unfed, (ii) inseminated/unfed, (iii) virgin/blood-fed and (iv) inseminated/blood-fed. Analysis of variance of two factors (2-way ANOVA) was performed to determine the significance of the effect of insemination, blood-feeding and the interaction of these two factors on the expression of the clock genes investigated. The results demonstrate blood-feeding alone is the only factor to cause consistent and significant impact on the gene expression. Therefore, for the graph representation, we joined the virgins and inseminated females and plotted only the difference between blood-fed and unfed status (Fig. 1 , Table I). Its effect on the body was significant for all four genes and significant for per and cyc and borderline for tim in the head (Table I). Fig. 1 shows that in all cases a reduction in clock gene expression was observed following blood-feeding.

RNAi knockdown of tim expression and its effects on activity behaviour - We conducted injections of in vitro synthesised tim dsRNA in adult Ae. aegypti females and monitored the effect of this intervention on the pattern of the mosquitoes activity/rest behaviour under two dif- ferent conditions, LD and DD. The efficacy of the RNAi in reducing tim expression was evaluated in head and body of mosquitoes collected on the fourth day after the dsRNA injection using qRT-PCR. A significant reduction in tim expression was detected in both heads and bodies in the two experiments (Fig. 2).

The graph of locomotor activity depicted in Fig. 3 shows similar patterns between dstim and dsLacZ, but with different levels of activity during seven days in LD. Under LD cycles, we expect an activity pattern with 24 $\mathrm{h}$, which represents a proper entrainment of mosquitoes to the LD regime. However, we were unable to observe any significant differences in the proportion of rhythmic individuals between $d s L a c Z$ and $d s t i m$ groups. Though, tim reduction decreases power levels (Table II). The power of the rhythm is usually defined as the amplitude from the top of the peak to the confidence level in the $\chi 2$ periodogram (Liu et al. 1991) and it is used as a parameter to measure the strength of the rhythm in a given environmental cycle. Thus we can assume that the power reduction in dstim mosquitoes is a consequence of a deficient light entrainment in this group.

In another experiment, after two days in LD the mosquitoes were submitted to five days in DD (Fig. 4A). In $\mathrm{DD}$, the number of rhythmic individuals diminishes equally between the two groups (Table II). Nevertheless, we observed a dramatic decrease in the activity levels in the dstim group in the second DD day, which corresponds to the fourth day after injection. After that, there is a gradual increase in the activity levels, but with a delayed phase shift as compared to the $d s L a c Z$ group. Also, there is a significant difference in the free running period between the groups (Table II), probably as a result of a great variety between individuals from the dstim group, which is not observed in the $d s L a c Z$ mosquitoes (Fig. 4B).

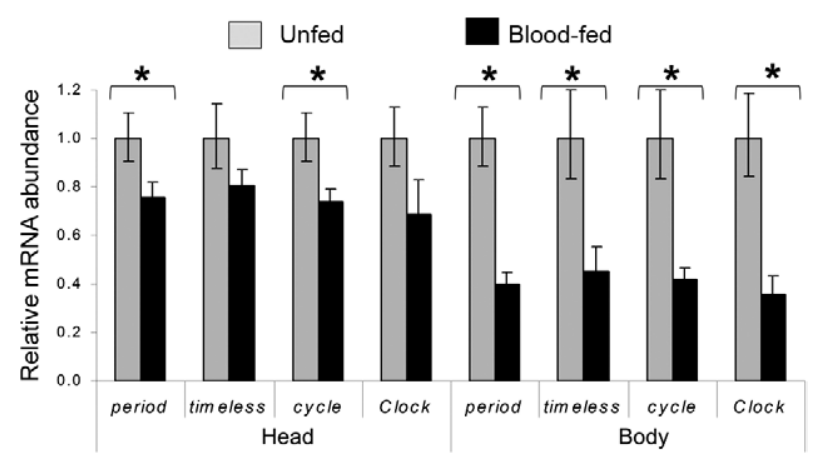

Fig. 1: expression of clock genes period, timeless, cycle and Clock relative to the constitutive gene rp49 in the head and body of Aedes aegypti females either fed or unfed on blood. Error bars show standard error. Asterisks indicate when RNA expression is significantly different between groups of blood-fed females and control (unfed). Since the statistics demonstrate blood-feeding is the only relevant factor in the analysis, for the graph representation, we joined the virgins and inseminated females and plotted only the difference between blood-fed and unfed status. For details on statistic results see Table I. 


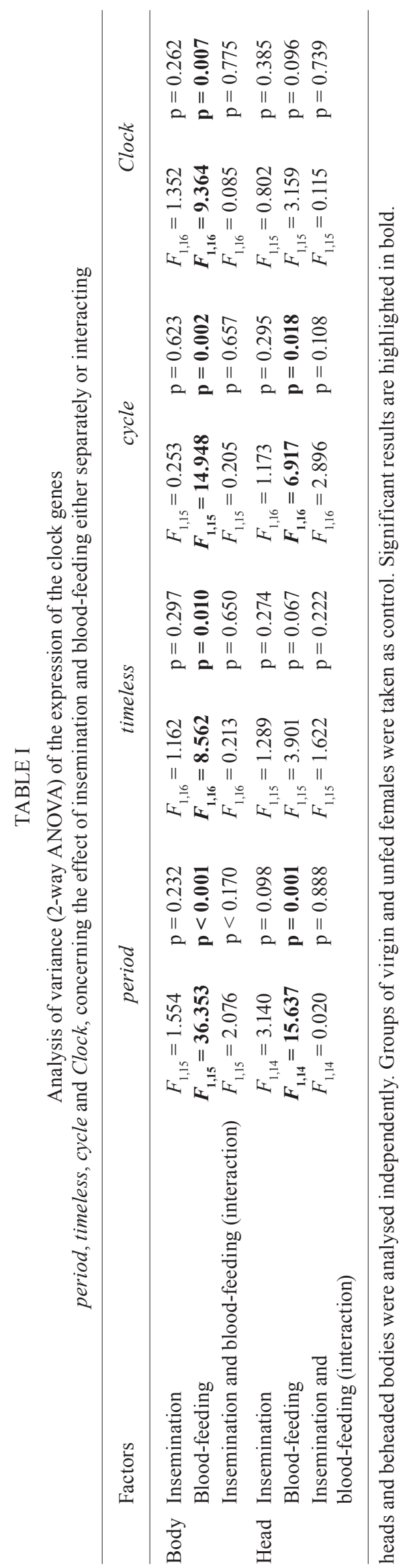

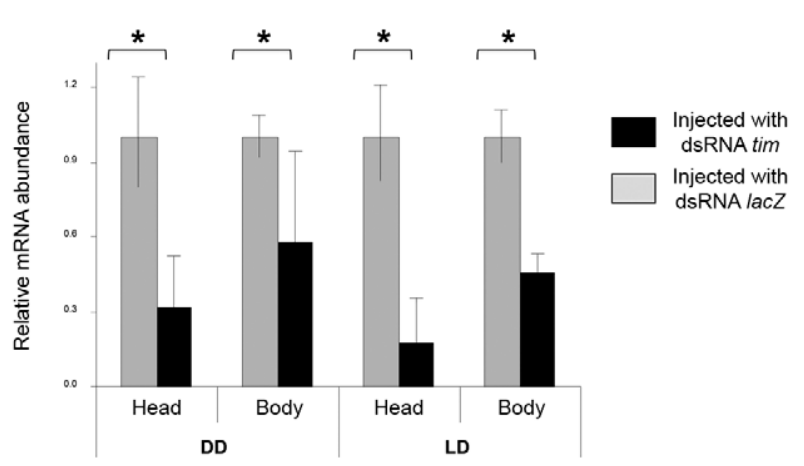

Fig. 2: expression of timeless relative to the constitutive gene rp 49 in the head and body of Aedes aegypti females injected with either timeless (tim) or lacZ double-stranded RNA (dsRNA). The highest value between these two is used as reference. A $t$ test was used to compare dstim and $d s L a c Z$ in each condition and all presented significant differences $(\mathrm{p}<0.05)$, marked by asterisks. Statistics indicates that concerning gene expression results, there are no differences between the two independent experiments (DD: constant darkness; LD: light/dark cycles). It also indicates only the fourth day after the injection with the dsRNA has significant change in gene expression on the group injected with dstim relative to the control group injected with dsRNA lacZ (ANOVA, $F_{158}=9.708 ; \mathrm{p}<0.003$ ). Therefore, this figure depicts only the statistically significant result of the fourth day after injections of both experiments. The greater reduction in tim expression observed in the head relative to the body specifically on the fourth day after injection had also been proven significant (ANOVA, $F_{1.58}=$ 8.226; $\mathrm{p}<0.006$ ). Error bars show standard error.

\section{DISCUSSION}

There is a wide interest in the behaviour of bloodsucking insects, due not only to the distress they cause to humans and other animals, but predominantly due to their role in the transmission of disease, with mosquitoes one of the most important groups in this regard. A particularly interesting aspect of the behaviour of haematophagous insects is the change observed in females according to their physiological state. For example, it has long been known that insemination, blood-feeding and the interaction of these two factors cause changes in the pattern of flight activity and rest behaviour in female mosquitoes (Jones \& Gubbins 1977, 1978, Jones 1981, Rowland 1989, Clements 1999). The explanation could rely on the ecological needs of each species. In nature, Anopheles mosquitoes generally mate in swarms and then females go chasing a blood meal. Interestingly, it has been shown for An. gambiae and An. stephensi that after mating, females change the profile of the flight activity and rest behaviour such that they no longer perfectly overlap with the males' pattern, as is the case for virgin females. Instead, they switch to a mode of persistent activity throughout the night that would favour their encounter with a source of blood meal. When inseminated and blood-fed, the female doesn't need to do much else but develop its eggs and shows almost no activity at all for two days. At which time it becomes active again to proceed with egg laying and the next search for a blood meal (Jones \& Gubbins 1978, Rowland 1989, Hawkes et al. 2012). However, the blood-feeding alone 


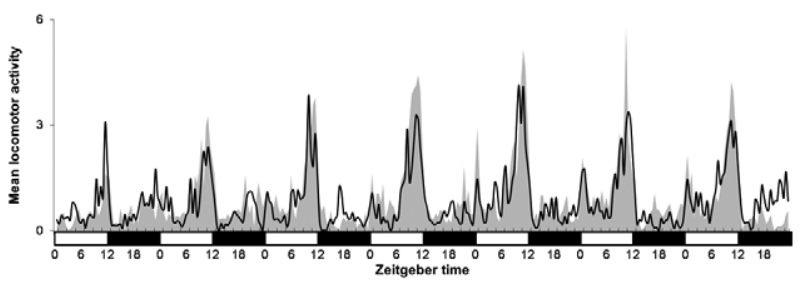

Fig. 3: activity/rest behaviour of two groups of Aedes aegypti female after the injection of double-stranded RNA (dsRNA) for seven days in light-dark cycles (LD). One group was injected with timeless dsRNA (black line) and the other with lacZ dsRNA (grey area), used as control. White horizontal bars indicate lights-on and black horizontal bars indicate light-off. Graphics depict average activity according to William's mean (Haddow 1960). Readings on the 30 min after the transitions LD and dark-light were interpolated to smooth startle responses.

does not seem to alter the flight activity pattern of virgin females, which continues to overlap with the males' activity, favouring the encounter for mating (Rowland 1989, Clements 1999). On the other hand, Ae. aegypti has different ecological needs from those of Anopheles species. They mate close to where the source of blood meal can be found (Jones 1981, Clements 1999). After mating, females do not need to be active for long periods in order to find a source of blood meal and in fact, they show a strong reduction in the overall activity after insemination. When mated and blood-fed, the activity of Ae. aegypti females is drastically suppressed, as seen in Anopheles, while blood-feeding alone causes only mild effects in the activity pattern (Jones 1981, Clements 1999, TN Lima-Camara, unpublished observations).

Despite an abundance of information on the behavioural and ecological observations, not much is known about the molecular mechanism of the circadian clock in mosquitoes. The expression pattern of the main clock genes in Ae. aegypti was previously described (Gentile et al. 2006, 2009) and shown to present a robust cycling expression, similar to Drosophila, with the exception of cyc expression. Even less is known about the molecular changes in clock genes triggered by insemination or blood-feeding. One of our aims in this study was to in- vestigate the impact of the blood meal on the expression of clock genes. Blood-feeding behaviour has been shown to be under circadian clock control (Das \& Dimopoulos 2008) and it is possible that this behaviour is not only regulated by, but also feeds back into, the circadian clock. This question has been addressed before in a study on the sandfly vector of leishmaniasis, Lu. longipalpis. The authors show $\sim 40 \%$ reduction in the overall activity as a result of blood intake (Meireles-Filho et al. 2006) and discuss that this reduction could be driven by the central pacemaker. It is known that two genes acting in haeme metabolism, $\delta$-aminolevulinate synthase and haemeoxygenase cycle in anti-phase in Drosophila heads (Ceriani et al. 2002). So it is possible that the oxidative stress caused by the blood meal could directly interfere with the regulation of the clock genes, causing an impairment on CLK's ability to bind DNA and driving the observed reduction in the expression of per and tim after blood intake. Our observations on Ae. aegypti reinforce this idea, since we show here that blood-feeding, and not insemination, is capable of causing a down regulation in the expression of the canonical clock genes per, tim, cyc and $C l k$ in the head and body of the females of this mosquito species (Fig. 1, Table I). In addition, $c y c$ regulation is under CLK control (Gentile et al. 2009) and it is also known that haeme selectively regulates the REV-ERB targets in mammals, including BMAL1, the Drosophila cyc orthologue gene (Raghuram et al. 2007). In mosquitoes, the Rev-ERB orthologue e75 is a nuclear receptor essential for haeme functions in the fat body (Cruz et al. 2012). These findings together suggest that two pathways may be acting together to reduce $c y c$ expression.

The central control of the circadian clock lies in a group of brain neurons called the clock neurons. While head extracts will not exclusively contain mRNA from clock neurons, they will closely reflect the overall expression pattern of clock genes in the central clock and for this reason are typically used as a proxy for the central clock molecular profile (Hardin 2011). Our results show that the blood meal is responsible for a significant reduction in the expression of per and $c y c$ in the head of females and a borderline reduction for tim and Clk (Fig. 1, Table I). One explanation for the borderline result with

TABLE II

Locomotor activity rhythms of Aedes aegypti in constant darkness injected with $d s L a c Z$ and $d s t i m$

\begin{tabular}{llcccc}
\hline Mosquitoes injected with & $\begin{array}{c}\text { Total } \\
\text { (n) }\end{array}$ & $\begin{array}{c}\text { Rhythmic mosquitoes } \\
(\%)\end{array}$ & $\begin{array}{c}\text { Period } \\
(\text { mean } \pm \text { SD }) / h\end{array}$ & $\begin{array}{c}\text { Power } \\
(\text { mean } \pm \text { SD) }\end{array}$ \\
\hline LD & dsLacZ & 23 & 79.3 & $24.23 \pm 0.11$ & $116.80 \pm 17.58$ \\
& dstim & 27 & 90 & $24.11 \pm 0.23$ & $81.68 \pm 9.93^{b}$ \\
DD & dsLacZ & 30 & 33.3 & $22.5 \pm 0.8$ & $37.72 \pm 6.10$ \\
& dstim & 27 & 33.3 & $25.25 \pm 1.0^{c}$ & $30.46 \pm 5.81$
\end{tabular}

$a$ : the power of the rhythm was defined as the amplitude from the peak to the cut-off line $(\alpha=0.05)$ in the $\chi^{2}$ periodogram (Liu et al. 1991); $b: t$ test, borderline statistical significance $(\mathrm{p}=0.07) ; c: t$ test, $\mathrm{p}<0.05$; DD: constant darkness; dsLacZ: double-stranded RNA (dsRNA) for LacZ; dstim: dsRNA for timeless; LD: light/dark cycles; SD: standard deviation. 
the latter two clock genes could lie in the fact that these genes have the lowest amplitude in their circadian fluctuation in the head of Ae. aegypti (Gentile et al. 2009), making it harder to detect a statistically significant reduction in their expression levels.

In the case of the clock genes' down-regulation in the body (Fig. 1, Table I), this reflects the state of the peripheral clocks. It is not possible to infer if it is caused by the down-regulation of the central clock or by a general mechanism responsible for the broader gene downregulation observed after a blood meal (Ribeiro 2003) or by both. Irrespective, the effect on the peripheral clock may be relevant to the observed output behaviour downstream from the central clock.

Peripheral clocks have already been shown to be important and sometimes even sufficient for maintaining some circadian aspects of behaviour and/or physiology (Hall 2005). A major component of the peripheral clock in insects is the fat body. The fat body is an insect analogue of the vertebrate liver and adipose tissue that also functions in other physiological processes such as innate immunity, detoxification and reproduction (Leclerc \& Reichhart 2004, Lazareva et al. 2007). A Drosophila microarray analysis on fat bodies along a circadian day identified several rhythmically-expressed genes that are controlled by this organ, including five cytochrome $\mathrm{P} 450$ genes and eleven oxidoreductase genes (Xu et al. 2011), necessary for haeme metabolism. So, it is possible to speculate that blood-feeding affects the insects' peripheral clock via fat bodies. On the other hand, the reduction observed in the body could relate more to the non-clock related functions these genes have in peripheral tissues. As discussed previously, mating seems to be the most relevant event in terms of the modulation of behaviour in Ae. aegypti (Jones 1981, Clements 1999). Ae. aegypti females are usually inseminated only once by males because accessory gland substances transferred from males make mated females refractory to subse- quent inseminations (Craig Jr 1967, Gillott 2003).

The evidence from Drosophila studies reinforces mating as the crucial event that modulates behaviour in females through changes at the molecular level [reviewed in Greenspan and Ferveur (2000)]. In mosquitoes, the molecular mechanism could be similar and some groups had been trying to investigate this. For example, it has been shown for An. gambiae that male accessory glands express genes that could potentially work as sex peptide-like modulators (Dottorini et al. 2007) and that mating triggers a wide range of gene expression changes (Rogers et al. 2008). However, we could not detect any significant change in clock gene expression in Ae. aegypti females in response to mating (Table I). We cannot discard the possibility that insemination alone is able to affect the clock gene expression in a subset of clock neurons or peripheral clocks. If the alteration in the gene expression is restricted, it could be undetectable with the strategy we adopted, using RNA extract from whole heads or beheaded bodies. Alternatively, the molecular changes following mating might not involve the circadian clock at all, but other behavioural controlling genes downstream from the clock. This hypothesis could only be tested when more information about the genes controlling output behaviour is available.

Understanding in detail the behaviour of female mosquitoes that are vectors of disease is crucial for the epidemiology of the parasites/virus they transmit (Clements 1999). It has been known for a long time, both in the field and in the laboratory, that females in different physiologic states will show different patterns of circadian activity (Jones \& Gubbins 1977, 1978, Jones 1981, Lima-Camara et al. 2011). We know, for example, that dengue virus infected females show an increase in their locomotor activity (Lima-Camara et al. 2011) and avidity for blood (Maciel-de-Freitas et al. 2013). In a simplified mathematical model, if we assume an increase in the biting rate of infected Ae. aegypti, the number of

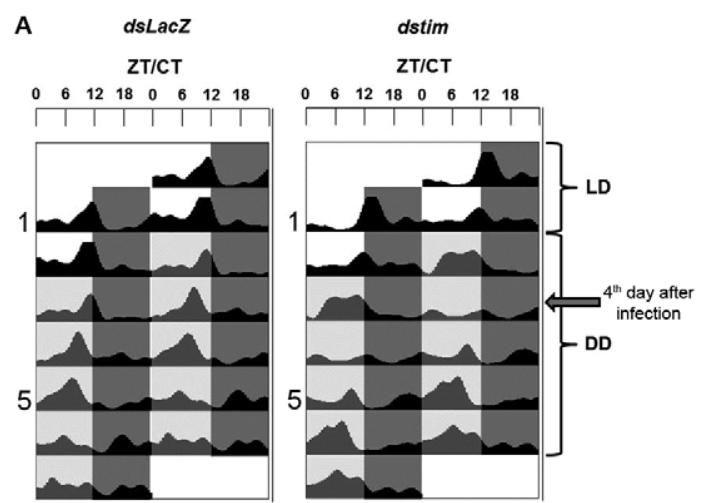

B

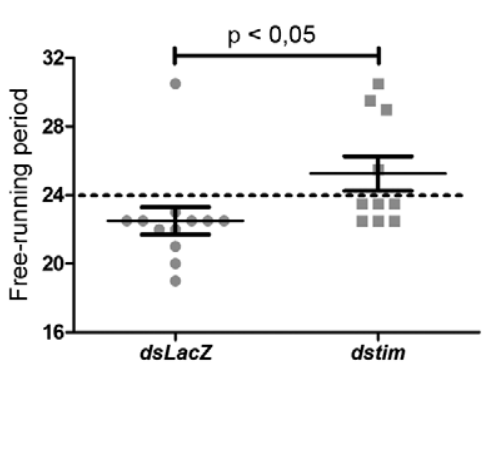

Fig. 4: locomotor activity/rest behaviour of two groups of Ae. aegypti female after the injection of double-stranded RNA (dsRNA) for two days in light-dark cycles (LD) followed by five days in constant darkness (DD). A: double-plotted actograms of representative mosquitoes injected with $d s L a c Z$ and dstim. The data was smoothed (Gaussian smoothing) using ActogramJ to reduce the noise and improve the visualisation of actograms. The shaded dark grey areas indicate dark phase and light grey areas indicate the subjective days in DD. The arrow shows a reduction of activity in dstim mosquitoes on the fourth day after the injection; B: mean \pm standard error of the means (lines) and individual mosquito (dslacZ, circles; dstim, squares) free running period length (hours). Statistical analysis by $t$ test show a difference between $d s l a c Z$ and $d s t i m$ ( $\mathrm{t}=$ $2.158 ; \mathrm{p}<0.05)$. A dotted horizontal line marks the $24 \mathrm{~h}$ value of $\mathrm{y}$ axis. CT: circadian time; ZT: Zeitgeber-time. 
primary and secondary infections also increases (Luz et al. 2011). However, the molecular mechanism behind this control has never been explored. We aimed in this paper to investigate the consequences of different physiologic states of female Ae. aegypti on the expression of the clock genes, fundamental in the control of the circadian rhythms, but further experiments are still needed to address this question.

Another aim of this work was to test the assumption of cause and effect in relation to clock gene expression and circadian behaviour. This assumption is based on what is known from Drosophila (Hall 2005, Hardin 2011), but testing this in Ae aegypti presents challenges due to limitations in the available genetic tools. We therefore chose to perform RNAi assays to knockdown tim and correlate the reduced expression with any circadian behavioural changes. The choice of tim was based on the sensitivity of the clock to a decrease in TIM protein concentrations caused by light-triggered degradation, resulting in a resetting of the whole system (Hall 2005, Hardin 2011). Results from Das and Dimopoulos (2008), who used a similar dsRNA injection technique, found a link between tim expression and blood-feeding behaviour.

Comparisons between TIM protein sequences in different insect species and Ae. aegypti (Danbara et al. 2010, Kamae \& Tomioka 2012) indicates that although the functional domains a reasonably similar, TIM may play distinct roles on the circadian clock of each species. Recent studies on the firebrat Thermobia domestica showed that Td'tim knockdowns (using dsRNA) abolished the locomotor rhythm in most of the treated firebrats for more than 30 days. In contrast, experiments using RNAi in the Orthoptera Gryllus bimaculatus (cricket) showed that Gb'tim dsRNA was able to reduce tim levels significantly, but was not sufficient to disrupt the circadian locomotor activity in DD. Despite not losing rhythmicity, the free running period is significantly shorter than control crickets injected with DsRed2 (Danbara et al. 2010).

Our results show in independent experiments that tim gene silencing was significant on the fourth day after injection (Fig. 2). This effect seems to influence the levels of locomotor activity in DD, four days after the injection (Fig. 4A). This reduction of tim could also promote longterm behaviour effects in both regimes. In fact, tim RNAi introduces changes that resemble the effects observed in the tim $^{0}$ mutant in Drosophila (Sehgal et al. 1994), since these mutants have rhythmic patterns of activity in LD, but are arrhythmic in DD. In LD, the increased activity of dstim in Ae. aegypti suggests changes in the masking effect by light, possibly due to the mosquitoes becoming less sensitive to light than the control group. Also, the lower levels of power in dstim (Table II) imply a small impairment in their entrainment to light. Together these results suggest that in mosquitoes tim plays a role in light masking and entrainment, as similarly observed with Drosophila [reviewed in Hardin (2011)]. In addition, under $\mathrm{DD}$, we observed an endogenous period longer than $24 \mathrm{~h}$ for dstim (Table II), but this likely reflects a substantial individual variation in the free-running periods as detected by the periodogram analysis (Fig. 4B). In several groups of insects it has been observed under constant conditions (constant light or constant darkness) that one peak of activity often splits into two or more components that free run with different phases (short and long periods) (Saunders 2002). Thus, it is possible that the reduction of tim favours the occurrence of splitting patterns of activity. The average endogenous period observed for dstim mosquitoes might be the mean of individuals in DD with both components free running simultaneously, but only one of these components can be detected by a periodogram analysis (Fig. 4B).

Interestingly, dstim injection into adult Gryllus bimaculatus has also generated increased activity in LD and a decrease in amplitude in DD (Danbara et al. 2010), albeit this does not disrupt its endogenous period in DD. Thus, although it is still premature to say, tim may have similar roles in the clock of these two species, playing an important role in both the light entrainment and modulation masking in LD and in DD, as well as the proper coupling of oscillators, the fine-tuning of the endogenous period and the amplitude control of locomotor activity. Finally, our results on the silencing of tim show that it is possible to achieve gene silencing in the head of the mosquito by thorax injection of dsRNA, however we could only show mild effect on the behaviour. As a consequence, we did not pursue with the silencing of the other individual clock genes.

\section{ACKNOWLEDGEMENTS}

To Andrea Crisanti and his team, for training C Gentile in RNAi assays at Imperial College, London, UK, to Samira Chahad-Ehlers and Julian Gray, for critical reading of the paper, and to Robson Costa da Silva, for technical assistance. This paper is dedicated to the memory of Alexandre Afranio Peixoto, an outstanding friend and scientist.

\section{REFERENCES}

Ceriani MF, Darlington TK, Staknis D, Más P, Petti AA, Weitz CJ, Kay SA 1999. Light-dependent sequestration of TIMELESS by CRYPTOCHROME. Science 285: 553-556.

Ceriani MF, Hogenesch JB, Yanovsky M, Panda S, Straume M, Kay SA 2002. Genome-wide expression analysis in Drosophila reveals genes controlling circadian behavior. J Neurosci 22: 9305-9319.

Clements AN 1999. The biology of mosquitoes: sensory reception and behaviour, CABI Publishing, New York, 740 pp.

Consoli RAGB, Lourenço-de-Oliveira R 1994. Principais mosquitos de importância sanitária no Brasil, Fiocruz, Rio de Janeiro, 228 pp.

Craig Jr GB 1967. Mosquitoes: female monogamy induced by male accessory gland substance. Science 156: 1499-1501.

Cruz J, Mane-Padros D, Zou Z, Raikhel AS 2012. Distinct roles of isoforms of the heme-liganded nuclear receptor E75, an insect ortholog of the vertebrate Rev-erb, in mosquito reproduction. Mol Cell Endocrinol 349: 262-271.

Danbara Y, Sakamoto T, Uryu O, Tomioka K 2010. RNA interference of timeless gene does not disrupt circadian locomotor rhythms in the cricket Gryllus bimaculatus. J Insect Physiol 56: 1738-1745.

Das S, Dimopoulos G 2008. Molecular analysis of photic inhibition of blood-feeding in Anopheles gambiae. BMC Physiol 16: 8-23.

Dottorini T, Nicolaides L, Ranson H, Rogers DW, Crisanti A, Catteruccia F 2007. A genome-wide analysis in Anopheles gambiae mosquitoes reveals 46 male accessory gland genes, possible modulators of female behavior. Proc Natl Acad Sci USA 104: 16215-16220. 
Emery P, So WV, Kaneko M, Hall JC, Rosbash M 1998. CRY, a Drosophila clock and light-regulated cryptochrome, is a major contributor to circadian rhythm resetting and photosensitivity. Cell 95: 669-679.

Filipecki AT, Machado CJ, Valle S, Teixeira MO 2011. The Brazilian legal framework on the scientific use of animals. ILAR J 52: e8-e15.

Garver L, Dimopoulos G 2007. Protocol for RNAi assays in adult mosquitoes (A. gambiae). $J$ Vis Exp 5: 230

Gentile C, Meireles-Filho AC, Britto C, Lima JB, Valle D, Peixoto AA 2006. Cloning and daily expression of the timeless gene in Aedes aegypti (Diptera: Culicidae). Insect Biochem Mol Biol 36: 878-884.

Gentile C, Rivas GB, Meireles-Filho AC, Lima JB, Peixoto AA 2009. Circadian expression of clock genes in two mosquito disease vectors: cry2 is different. $J$ Biol Rhythms 24: 444-451.

Gillott C 2003. Male accessory gland secretions: modulators of female reproductive physiology and behavior. Annu Rev Entomol 48: $163-184$

Greenspan RJ, Ferveur JF 2000. Courtship in Drosophila. Annu Rev Genet 34: 205-232.

Hall JC 2005. Systems approaches to biological rhythms in Drosophila. Methods Enzymol 393: 61-185.

Hardin PE 2011. Molecular genetic analysis of circadian timekeeping in Drosophila. Adv Genet 74: 141-173.

Hardin PE, Panda S 2013. Circadian timekeeping and output mechanisms in animals. Curr Opin Neurobiol 23: 724-731.

Hawkes F, Young S, Gibson G 2012. Modification of spontaneous activity patterns in the malaria vector Anopheles gambiae sensu stricto when presented with host-associated stimuli. Physiol Entomol 37: 233-240.

Jones MDR 1981. The programming of circadian flight-activity in relation to mating and the gonotrophic cycle in the mosquito, Aedes aegypti. Physiol Entomol 6: 307-313.

Jones MDR, Gubbins SJ 1977. Modification of circadian flight activity in the mosquito Anopheles gambiae after insemination. $\mathrm{Na}$ ture 268: 731-732.

Jones MDR, Gubbins SJ 1978. Changes in the circadian flight activity of the mosquito Anopheles gambiae in relation to insemination, feeding and oviposition. Physiol Entomol 3: 213-220.

Kamae Y, Tomioka K 2012. Timeless is an essential component of the circadian clock in a primitive insect, the firebrat Thermobia domestica. J Biol Rhythms 27: 126-134.

Lazareva AA, Roman G, Mattox W, Hardin PE, Dauwalder B 2007. A role for the adult fat body in Drosophila male courtship behavior. PLoS Genet 3: e16.

Leclerc V, Reichhart JM 2004. The immune response of Drosophila melanogaster. Immunol Rev 198: 59-71.

Lehane MJ 2005. The biology of blood sucking insects, 2nd ed., Cambridge University Press, New York, 336 pp.

Lima-Camara TN, Bruno RV, Luz PM, Castro MG, Lourenço-deOliveira R, Sorgine MHF, Peixoto AA 2011. Dengue infection increases the locomotor activity of Aedes aegypti females. PLoS ONE 6: e17690.

Liu X, Yu Q, Huang Z, Zwiebel LJ, Hall JC, Rosbash M 1991. The strength and periodicity of D. melanogaster circadian rhythms are differentially affected by alterations in period gene expression. Neuron 6: 753-766.

Luz PM, Lima-Camara TN, Bruno RV, de Castro MG, Sorgine MH, Lourenço-de-Oliveira R, Peixoto AA 2011. Potential impact of a presumed increase in the biting activity of dengue-virus-infected Aedes aegypti (Diptera: Culicidae) females on virus transmission dynamics. Mem Inst Oswaldo Cruz 106: 755-758.

Maciel-de-Freitas R, Sylvestre G, Gandini M, Koella JC 2013. The influence of dengue virus serotype-2 infection on Aedes aegypti (Diptera: Culicidae) motivation and avidity to blood feed. PLoS ONE 8: e65252.

Meireles-Filho AC, Rivas GBS, Gesto JS, Machado RC, Britto C, de Souza NA, Peixoto AA 2006. The biological clock of an hematophagous insect: locomotor activity rhythms, circadian expression and downregulation after a blood meal. FEBS Lett 580: 2-8.

Pfaffl MW 2001. A new mathematical model for relative quantification in real-time RT-PCR. Nucleic Acids Res 29: e45.

Raghuram S, Stayrook KR, Huang P, Rogers PM, Nosie AK, McClure DB, Burris LL, Khorasanizadeh S, Burris TP, Rastinejad F 2007. Identification of heme as the ligand for the orphan nuclear receptors REV-ERBalpha and REV-ERBbeta. Nat Struct Mol Biol 14: $1207-1213$

Ribeiro JM 2003. A catalogue of Anopheles gambiae transcripts significantly more or less expressed following a blood meal. Insect Biochem Mol Biol 33: 865-882.

Rogers DW, Whitten MM, Thailayil J, Soichot J, Levashina EA, Catteruccia F 2008. Molecular and cellular components of the mating machinery in Anopheles gambiae females. Proc Natl Acad Sci USA 105: 19390-19395.

Rowland M 1989. Changes in the circadian flight activity of the mosquito Anopheles stephensi associated with insemination, bloodfeeding, oviposition and nocturnal light intensity. Physiol Entomol 14: 77-84.

Rund SS, Gentile JE, Duffield GE 2013. Extensive circadian and light regulation of the transcriptome in the malaria mosquito Anopheles gambiae. BMC Genomics 14: 218

Rund SS, Hou TY, Ward SM, Collins FH, Duffield GE 2011. Genomewide profiling of diel and circadian gene expression in the malaria vector Anopheles gambiae. Proc Natl Acad Sci USA 108: e421-e 430

Sandrelli F, Costa R, Kyriacou CP, Rosato E 2008. Comparative analysis of circadian clock genes in insects. Insect Mol Biol 17: 447-463.

Saunders DS 2002. Insect Clocks, 3rd ed., Elsevier Science, Amsterdam, $560 \mathrm{pp}$

Schmid B, Helfrich-Förster C, Yoshii T 2011. A new ImageJ plug-in "ActogramJ" for chronobiological analyses. J Biol Rhythms 26: 464-467.

Sehgal A, Price JL, Man B, Young MW 1994. Loss of circadian behavioral rhythms and per RNA oscillations in the Drosophila mutant timeless. Science 263: 1603-1606.

Stanewsky R, Kaneko M, Emery P, Beretta B, Wager-Smith K, Kay SA, Rosbash M, Hall JC 1998. The cryb mutation identifies cryptochrome as a circadian photoreceptor in Drosophila. Cell 95: $681-692$

Xu K, DiAngelo JR, Hughes ME, Hogenesch JB, Sehgal A 2011. The circadian clock interacts with metabolic physiology to influence reproductive fitness. Cell Metab 13: 639-654. 